\title{
"Everything is a Mess": How COVID-19 is Impacting Engagement with HIV Testing Services in Rural Southwestern Uganda
}

\author{
Matthew Ponticiello ${ }^{1} \cdot$ Juliet Mwanga-Amumpaire ${ }^{2} \cdot$ Patricia Tushemereirwe $^{2} \cdot$ Gabriel Nuwagaba $^{2} \cdot$ Rachel King $^{3}$. \\ Radhika Sundararajan ${ }^{4,5}$
}

Published online: 25 May 2020

○) Springer Science+Business Media, LLC, part of Springer Nature 2020

In southwestern Uganda, where HIV prevalence among sexually active adults is $7 \%$ [1], we have been conducting longitudinal research to improve uptake of HIV testing among communities who utilize informal health providers, such as traditional healers. On March 12, 2020, the first case of COVID-19 was described in eastern Africa [2]. Shortly thereafter, Ugandan President Museveni enacted countrywide ordinances to mitigate the spread of the disease [3]. These restrictions included suspension of public gatherings, closure of schools and non-essential business, discontinuation of public transportation, and enforcement of a national curfew. At the time of these regulations, our study was conducting scheduled 90-day follow up interviews among participants referred for voluntary HIV testing at the District regional referral hospital. In this Note from the Field, we present data from 20 in-depth interviews conducted between April and May 2020 describing the impact of COVID-19 on uptake of HIV testing in southwestern Uganda. We believe the experiences of our participants may be representative of other rural, HIV-endemic regions of sub-Saharan Africa.

Radhika Sundararajan

ras9199@med.cornell.edu

Cornell University, Ithaca, NY, USA

2 Department of Pediatrics, Mbarara University of Science and Technology, Mbarara, Uganda

3 Global Health Sciences, University of California, San Francisco, San Francisco, CA, USA

4 Weill Cornell Center for Global Health, 402 East 67th Street, 2nd floor, New York, NY 10065, USA

5 Weill Cornell Department of Emergency Medicine, New York, NY, USA

\section{Qualitative Data Collection}

All participants were enrolled in the parent study 90-days prior to the date of their exit interview. They were aged $\geq 18$ years, received care from a traditional healer at time of enrollment, had not previously been diagnosed with HIV, and self-reported no HIV testing in the 12 months prior to enrollment. Interviews explored factors shaping utilization of voluntary HIV testing services, and were not originally intended to include COVID-19 as a topic of discussion. While reviewing interviews conducted after COVID-related ordinances were enacted, we noted that all participants mentioned COVID-19 as a significant disruptive factor in their lives.

All interviews included in this analysis were conducted between 1 April and 15 May 2020.

Interviews were conducted in the local language (Runyankole) and translated/transcribed into English for analysis, by two co-authors (PT, GN) fluent in both languages. Interviews were completed over the phone, and audio recorded with permission, to comply with social distancing protocols. Following an inductive, content-analysis approach [4], transcripts were reviewed and coded by two authors (MP, RS) to identify broad categories illustrating the impact of COVID19 on utilization of HIV testing resources, with representative quotes shown below.

\section{What we Learned}

Characteristics of study participants are shown in Table 1. Our qualitative results demonstrate that COVID-19 has negatively impacted engagement with HIV testing resources in two important ways: (1) COVID-restrictions prevent participants from accessing HIV testing services; and (2) COVIDrelated stigma discourages use of healthcare facilities where HIV testing services are located. 
Table 1 Characteristics of study participants

\begin{tabular}{ll}
\hline Participant characteristic & Distribution $(\mathrm{N}=20)$ \\
\hline Gender & Male $=10$, Female $=10$ \\
Age & Mean $=31.1(\mathrm{SD}=8.1)$ \\
Marital status & Married/Cohabiting $=12$ \\
Highest level of education & Primary school or less $=9$ \\
& Secondary school \& higher $=11$ \\
Household size & Mean $=4.2(\mathrm{SD}=1.8)$ \\
Religious affiliation & Christian $=17$, Muslim $=3$ \\
Ever received an HIV test & Yes $=17$ \\
Months since last HIV test at enrollment & Mean $=35.2(\mathrm{SD}=14.1)$ \\
Recruited from traditional healer specialty & Bonesetter $=15$, Herbalist $=5$ \\
\hline
\end{tabular}

\section{COVID-19 Restrictions Impede Access to HIV Testing Services}

Participants described that travel restrictions and closure of businesses have created significant barriers to accessing voluntary HIV testing services. Most residents of our study region rely on public transportation to reach hospitals and clinics, as they reside significant distances from healthcare facilities. In light of public transportation restrictions, people are unable to access healthcare located outside of walking distance. A participant describes her inability to reach HIV testing services at the district hospital:

[COVID-19] caused trouble to us because even if you have money, there are no means you can reach the hospital. All public transport-and even private vehicles-were restricted. So even if I get money now, I cannot go until the government allows public transport to operate. (Female, 28 years old).

Another participant explained the impact of COVIDrelated travel restrictions on his intent to receive voluntary HIV testing:

I worked so hard to save [money] for transportation that would take me to Mbarara Referral Hospital and [receive an HIV] test, but as I was preparing to go and test that is when the country was locked down due to COVID... There is no transport means. I cannot walk to the hospital. It is very far. (Male, 32 years old).

COVID-19 ordinances also mandated closure of public markets and non-essential businesses to minimize the spread of the virus within communities. Participants report resultant drop in household income, and subsequent inability to pay for healthcare services. As transportation restrictions impede access to free services provided at government-run facilities, participants can only obtain HIV testing at facilities located within walking distance.
These are typically private-often unlicensed-clinics which operate on a fee-for-service model. One participant explained:

Health services are provided free in the government facilities. But when accessing them in the private clinics, you have to have some money, which we do not have. (Male, 23 years old).

Another participant described how loss of income has affected his ability to receive HIV testing at one of the local, private clinics:

You see, since COVID started, most families no longer have any source of income and you cannot access healthcare for free ... when it comes to private health facilities, even seeing a doctor, you pay for it. So, it is very hard for us [to receive HIV testing], since we no longer work. (Male, 36 years old).

\section{COVID-Related Stigma Creates Reluctance to Visit Facility-Based HIV Testing Services}

COVID-stigma has been associated with those testing positive for the virus $[5,6]$, or those living and working in close proximity with COVID-19 patients [7]. Our participants describe numerous stigmatizing beliefs which underlie reluctance to seek care at formal healthcare facilities. They express fear of becoming infected through proximity to COVID patients, which motivates avoidance of voluntary HIV services, and healthcare services more broadly:

I fear to go to the hospital now because of Coronavirus. I don't want to risk it. Coronavirus patients are being attended from there. I fear to be infected. But when the situation stabilizes, I will go and get [HIV] tested. (Male, 25 years old).

People do not seek medical care from the hospital, fearing to be put under quarantine in case they are found to have COVID-19. Others fear to be exposed 
and get infected. So, people prefer staying in their homes even when they are sick. (Female, 27 years old).

In addition, some individuals report that they avoid healthcare facilities due to concern for receiving substandard medical care:

Health workers are reluctant to attend to you... they also fear to get infected especially if you have some signs that are related to COVID-19. (Male, 39 years old).

One participant summarized the broad impacts of COVID-19 on her family:

This situation is affecting us in all corners. My family no longer works. Their businesses were closed. We cannot access medical care because we fear to walk to the health facilities, and there are no means of transportation. We cannot take our goods to the markets [to sell]. So, everything is a mess. (Female, 28 years old).

\section{Implications for HIV Testing and Epidemic Control}

Uganda has made notable gains towards UNAIDS Fast Track targets [1, 8], but the COVID-19 pandemic threatens to undermine Uganda's progress by exacerbating existing barriers to HIV testing. For example, before COVID-19, HIV testing uptake was already lower in rural and impoverished regions of the country, and among men [9]. Distance to biomedical clinics has also been shown to decrease HIV service utilization in rural Uganda [10, 11]. Like much of sub-Saharan Africa, our study region is medically pluralistic, where people seek care from both formal and informal healthcare resources. We have previously described preference for traditional healing, sometimes rooted in mistrust of biomedicine [12]. The qualitative data presented here illustrate how COVID-19 is inhibiting access to and uptake of HIV testing services among rural Ugandans by underscoring existing structural barriers (lack of transportation, poverty, location of biomedical resources), and through the influence of new, stigmatizing beliefs surrounding COVID-19.

Many have raised the alarm that COVID-19 will undermine global advances towards HIV epidemic control through disrupting the cascade of care [13-15]. Indeed, we show that this pandemic impedes uptake of HIV testing, the critical entry point into HIV treatment and care [16]. However, findings from our study can inform effective and adaptive solutions to mitigate the deleterious impacts of COVID-19 in HIV endemic regions [17]. Strategies to decentralize HIV testing services among rural communities could mitigate the barriers we describe above. While mobile outreach services have had success in the past [18], these initiatives require significant investment and infrastructure, and may be difficult to implement in the setting of COVID-related social distancing protocols.

Instead, we propose the scale-up of HIV self-test distribution as a feasible, sustainable approach to increase access to HIV testing in the context of COVID-19 in sub-Saharan Africa. Given previously described barriers to self-testing [19], we recommend this initiative be carried out in collaboration with traditional healers, who live and practice in villages, and are highly regarded members of their communities [20]. While maintaining a safe distance, healers could be available to supervise clients' self-testing, aid with interpreting testing results, offer counseling and support following test results, encourage linkage to care for those testing positive, and distribute kits to those wishing to test at home. Finally, we suggest that COVID-stigma reduction initiatives will be critically important to combat growing fear and mistrust of biomedicine among rural populations. Addressing stigma and discrimination towards people recovering from COVID-19, and towards healthcare workers caring for COVID-19 patients, within these communities should be a priority component of COVID-recovery efforts.

\section{References}

1. PEPFAR. PEPFAR Uganda Country Operational Plan 2019. 2019. https://www.state.gov/wp-content/uploads/2019/09/Ugand a_COP19-Strategic-DirectionalSummary_public.pdf. Accessed 14 May 2020.

2. Kenya Ministry of Health. Kenya Coronavirus cases rise to four. 17 March, 2020. https://www.health.go.ke/kenya-coronaviru s-cases-rise-to-four-cs-health. Accessed 18 May 2020.

3. Home-COVID-19| Ministry of Health. 2020. https://www.healt h.go.ug/covid/. Accessed 15 May 2020.

4. Hsieh H-F, Shannon SE. Three approaches to qualitative content analysis. Qual Health Res. 2005;15(9):1277-88.

5. UNAIDS. Rights in the time of COVID-19: Lessons from HIV for an effective, communityled response. 2020. https://www.unaid s.org/en/resources/documents/2020/human-rights-and-covid-19. Accessed 15 May 2020.

6. Logie CH, Turan JM. How do we balance tensions between COVID-19 public health responses and stigma mitigation? Learning from HIV research. AIDS Behav. 2020;17(1):31-4.

7. World Health Organization. Mental health and psychosocial considerations during the COVID-19 pandemic 2020. https://apps. who.int/iris/bitstream/handle/10665/331490/WHO-2019-nCoVMentalHealth2020.1-eng.pdf. Accessed 16 May 2020.

8. UNAIDS. UNAIDS: Fast Track to end AIDS by 2030. 2015. https ://www.unaids.orgenresourcesdocumentsJCWADreport. Accessed 14 May 2020.

9. Ugandan Ministry of Health. Uganda Population-based HIV Impact Assessment (UPHIA) 2016-2017. 2019. httpwww.afro. who.intsitesdefaultfiles-UPHIAUgandafactsheet.pdf. Accessed 16 May 2020.

10. Siedner MJ, Lankowski A, Tsai AC, et al. GPS-measured distance to clinic, but not selfreported transportation factors, are associated with missed HIV clinic visits in rural Uganda. AIDS. 2013;27(9):1503-8. 
11. Mayer CM, Owaraganise A, Kabami J, et al. Distance to clinic is a barrier to PrEP uptake and visit attendance in a community in rural Uganda. J Int AIDS Soc. 2019;22(4):e25276.

12. Sundararajan R, Mwanga-Amumpaire J, King R, Ware NC. Conceptual model for pluralistic healthcare behaviour: results from a qualitative study in southwestern Uganda. BMJ Open. 2020;10(4):e033410.

13. Wilkinson L, Grimsrud A. The time is now: expedited HIV differentiated service delivery during the COVID-19 pandemic. J Int AIDS Soc. 2020;23(5):e25503.

14. Ridgway JP, Schmitt J, Friedman E, et al. HIV care continuum and COVID-19 outcomes among people living with HIV during the COVID-19 Pandemic, Chicago. IL AIDS Behav. 2020;63(47):1113-23.

15. Sun S, Hou J, Chen Y, Lu Y, Brown L, Operario D. Challenges to HIV care and psychological health during the COVID-19 Pandemic among people living with HIV in China. AIDS Behav. 2020;20(1):22.

16. World Health Organization. Consolidated guidelines on HIV testing services. 2015. https://www.who.int/hiv/pub/guidelines/hivtesting-services/en/. Accessed 15 May 2020.
17. Bali S, Dhatt R, Lal A, et al. Off the back burner: diverse and gender-inclusive decisionmaking for COVID-19 response and recovery. BMJ Glob Health. 2020;5(5):e002595.

18. Chamie G, Kamya MR, Petersen ML, Havlir DV. Reaching 90-9090 in rural communities in East Africa: lessons from the sustainable East Africa research in community health trial. Curr Opin HIV AIDS. 2019;14(6):449-54.

19. Harichund C, Moshabela M. Acceptability of HIV selftesting in Sub-Saharan Africa: scoping study. AIDS Behav. 2018;22(2):560-8.

20. Homsy J, King R, Balaba D, Kabatesi D. Traditional health practitioners are key to scaling up comprehensive care for HIV/AIDS in sub-Saharan Africa. AIDS. 2004;18(12):1723-5.

Publisher's Note Springer Nature remains neutral with regard to jurisdictional claims in published maps and institutional affiliations. 